Terpenoids in the surface soils from different ecosystems on the Tibetan Plateau YAN BAI $^{1,2}$ *, QINGHU CHEN ${ }^{1,3}$, XIAOMIN FANG ${ }^{1,2,3}$, ChIHAO CHEN ${ }^{1,3}$, XIAOMING LIU ${ }^{1}$

${ }^{1}$ CAS Key Laboratory of Continental Collision and Plateau Uplift, Institute of Tibetan Plateau Research, Chinese Academy of Sciences

${ }^{2}$ CAS Center of Excellence for Tibetan Plateau Earth Sciences, Chinese Academy of Sciences

${ }^{3}$ University of the Chinese Academy of Sciences

\title{
Introduction
}

This project identified 46 terpenoids isolated from 31 surface soil samples collected along an altitudinal vegetation gradient on the eastern slopes of Mountain Gongga, eastern Tibetan Plateau (TP), an area with a temperate monsoonal climate, compared with 29 surface soils taken from the northern slopes of the Kunlun Mountains, a region characterized by montane desert, meadow and alpine steppe.

\section{Results and significance}

Various polycyclic triterpenoids of the taraxerane, oleanane, friedelane, ursane, lupane, glutinane and lanostane series were present in varying relative abundances, and generally corresponded with those derived from the lipids extracted from the overlying vegetation as part of this study, or reported by other studies. We also uncovered a dozen new triterpenoids from soils. Due to their absence across the Kunlun Mountains (with the exception of the presence of taraxeranes in only a few of the investigated samples), the presence of these highly specific compounds in sediment could therefore be of significance in palaeo-vegetation reconstructions and help determine the time in which trees (forests) became established.

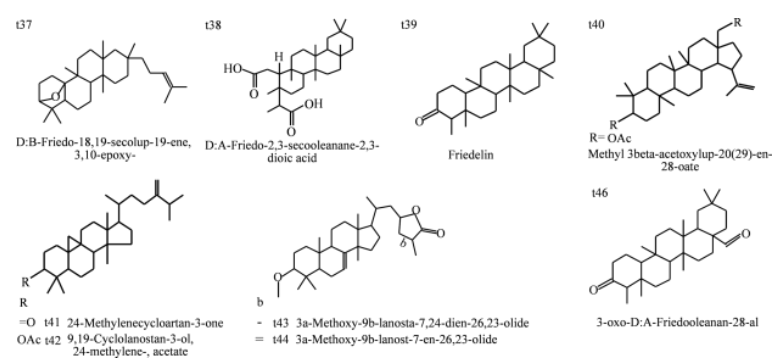

Fig 1. Structures of the new triterpenoids tentatively identified in this study. 